IRA-International Journal of Management \&

Social Sciences

ISSN 2455-2267; Vol.08, Issue 03 (September 2017)

Pg. no. 256-265

Institute of Research Advances

http://research-advances.org/index.php/RAJMSS

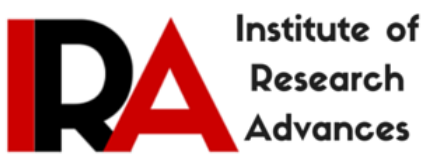

\title{
A Study of New Age Learning Network for Business Impact in Indian Service Industry with Specific Reference to Information Technology Sector
}

\author{
Prasad G. Akerkar \\ Research Scholar, Sydenham Institute of Management Studies, Research and Enterprise Education \\ (SIMSREE), Mumbai, India.

\section{Dr. R. K. Srivastava} \\ Professor and Head, Sydenham Institute of Management Studies, Research and Enterprise Education \\ (SIMSREE), Mumbai, India.
}

Type of Review: Peer Reviewed.

DOI: http://dx.doi.org/10.21013/jmss.v8.n3.p4

How to cite this paper:

Akerkar, P.G., Srivastava, R.K. (2017). A Study of New Age Learning Network for Business Impact in Indian Service Industry with Specific Reference to Information Technology Sector. IRA-International Journal of Management \& Social Sciences (ISSN 2455-2267), 8(3), 256-265. doi:http://dx.doi.org/10.21013/jmss.v8.n3.p4

(C) Institute of Research Advances.

\section{(cc) BY-NC}

This work is licensed under a Creative Commons Attribution-Non Commercial 4.0 International License subject to proper citation to the publication source of the work.

Disclaimer: The scholarly papers as reviewed and published by the Institute of Research Advances (IRA) are the views and opinions of their respective authors and are not the views or opinions of the IRA. The IRA disclaims of any harm or loss caused due to the published content to any party.

Institute of Research Advances is an institutional publisher member of Publishers Inter Linking Association Inc. (PILA-CrossRef), USA. The institute is an institutional signatory to the Budapest Open Access Initiative, Hungary advocating the open access of scientific and scholarly knowledge. The Institute is a registered content provider under Open Access Initiative Protocol for Metadata Harvesting (OAI-PMH).

The journal is indexed \& included in WorldCat Discovery Service (USA), CrossRef Metadata Search (USA), WorldCat (USA), OCLC (USA), Open J-Gate (India), EZB (Germany) Scilit (Switzerland), Airiti (China), Bielefeld Academic Search Engine (BASE) of Bielefeld University, Germany, PKP Index of Simon Fraser University, Canada. 


\begin{abstract}
Service Sectors in India is undergoing major transformation phase in today's time. In order to achieve sustainable profitable growth, this sector is facing key competitive challenges such as operational efficiency, delivery productivity, global customer satisfaction and talent development \& retention across their delivery centers. Training, Learning \& Development (L\&D) plays a significant role in these organizations by providing cutting edge leadership in technology \& business management. It's therefore very important to accomplish and measure the effectiveness and efficiency of $L \& D$ for real life business application and building learning assets for such organizations. Interestingly, India has one of the largest young workforce in the world, with over 65 $\%$ of its population under the age of 35. Today, Indian service sector employ about 3-4 generations at a time with generation Y alias millennial constituting about $70 \%$ of the total workforce. They have varied social demographic, have changing interests in diverse fields, and are innovative, creative \& restless in nature. With their diverse learning\& performing styles, varied expectations and challenges, it's important to build a contemporary learning network \&ecosystem that is tech-savvy, supports social and collaborative learning and provides linkages to growth of individuals, groups and organizations, thus leading to improved business performance at holistic level. The purpose of this paper, therefore, is to examine this significant variable of $L \& D$ ecosystem constituting to its effectiveness for business outcome, ways of formal and informal learning channels that resonate most with these next gen employees, being part of a multi-generation workforce today.
\end{abstract}

Keywords: Training effectiveness, Next generation learning, Learning Ecosystem, Business Results, IT Organizations, India

\title{
Introduction:
}

India today is the favourite destinations for Information Technology (IT) outsourcing and the hub for developing low cost, good quality IT solutions for global markets. According to IMF study on anchoring growth in emerging markets and developing economies, technology plays a significant role in resource capitalization, productivity gains and sustainable economic growth. . Despite the slowdown that had engulfed technology spending worldwide, this sector continued to grow at a robust rate with CAGR of around 13-15\% during last decade. Indian Information Technology-Business Process Management (IT-BPM) sector revenue recorded an impressive growth of 13\% from USD 130 billion in FY2014 to USD 160 billion in FY2016 with IT services revenue share alone at USD 75 billion. Interestingly, today while the export segment share account for about $68 \%$ of total revenue, the domestic segment is also growing steadily on account of central government's programs on "Digital India", "Make in India" and "Start-up India". However, currency volatility, macroeconomic headwinds and obstacles for quick adaption to changing technologies are likely to pull down growth of Indian IT \& BPM services exports to around 10-11 \% for FY17 \& FY18 (Source: Nasscom Strategic Review Report, February 2016). It's therefore necessary to examine the ways and means that can rapidly elevate this industry from the perspective of productivity and operational efficiency, eventually achieving the revenue, profit and growth targets on sustainable basis. Researchers, practitioners and industry experts believe that Learning and Development (L\&D) is one such field that can play a significant role to provide required impetus for sustainable growth. Therefore, continuous training \& learning is an absolute essential dimension of this industry for achieving profitable growth and sustainability. This leads to higher importance of analysing training effectiveness and its impact on business results in Indian IT organisations. It's therefore highly imperative to study the factors contributing the training \& learning effectiveness, how and to what extent they can enhance performing abilities of employees to deliver results and eventually which components of business results would have potential impact due to such enhanced ability to perform.In today's changing times, when learners are primarily belong to the next generation era, it's essential that the learning ecosystem is designed and developed to suit their changing needs, inter-alia, that of the organization. It should have better user experience and appeal, provides easy help to these next gen learners to access, assimilate, attain and retain the learning for developing profound capabilities and eventually apply them on the ground to make a change. Theresearch paper, therefore, focuses on this important factor and discusses how a conducive learning ecosystem can be built to help the employees of business corporates, governments, trade \& commerce equally well and leverage the same for the higher purposes.

\section{Statement of Opportunity}

Indian Information Technology (IT) companies classically deliver its cutting edge technological services and solutions to its worldwide customers through the network of global delivery centres and diverse global workforce. Human Resources therefore play the most significant part towards the success of end-to-end talent supply chain management. Their knowledge, skill and attitude determine their global competitiveness and hence their abilities to build a stronger learning value to deliver the promises. However, this sector is facing some daunting challenges in this area which primarily 
include low operational efficiency and burgeoning skill gaps in technological and functional areas. Today, majority of IT employees who operate at the bottom of the pyramid are generally between the age group of 21 and 35 . Their capabilities to learn next\& diverse skills, retain the learning in active memory and eventually apply them on the job effectively, on continuous basis, have been a major challenge to organisations and management experts. This sector is often entangled with challenges like how to respond to the changing situation quickly and effectively? How to earn customer confidence and trust through flexibility, learning on-the-go and delivering higher customer value? How to grow business by increasing revenue, controlling cost and managing operating profits, in addition to building learning and delivery assets for future readiness and excellence?

According to Andrew Calkins and Kristen Vogt (2013), next generation learning isn't about educating the next generation of students, but about engaging with today's students through "next gen" teaching and learning designs that promise significantly higher achievement for many more students than current-generation approaches have been able to generate. Next generation learning is catalyzed and informed by:

- A deepened understanding of learning: how, where, and why students (and people of all ages) learn most effectively

- A deepened understanding of learners: what's required to engage and meet students' complex, individual needs, especially given the vibrant diversity of the nation's current and future population of young people

- The recognition that the world has changed: so thoroughly, in fact, that it requires a much higher level of achievement for much higher percentages of students

Continuous learning, therefore is the mantra and forms a valuable part of business strategy, as technology is changing rapidly and the need of doing more with less is critical than ever. Employee preferences are changing, business success parameters are changing, customer expectations are changing and fortunately, so is the technological support to achieve a common goal. L\&D in Indian IT organisations therefore can play a crucial role in achieving these objectives through a thoughtful balance. This will lead to real effectiveness of learning and training, including supplementary benefits such as helping employees to build their own individual development plans (IDP) \& career progression, enhancing intrinsic motivation thus reducing attrition and improving productivity etc. Importantly, there is also a growing need to look at learning and development function, not as a cost driver but more so as a value provider. It has been experienced that, in order to address the growing technology competency needs, continuous skill up-gradation is essential through upskilling, cross-skilling and multi-skilling. While traditionally, Instructor led classroom training was the primary medium of training, with advent of technology and changing preferences of employees, business and customers, the IT companies replacing this traditional model with the next gen models including e-learning, group learning, virtual learning, social learning, collaborative learning and learning at your own pace, place and device etc. The onus of learning is now quickly getting shifted from trainer to learner for a pull model rather than a push model. This win-win is here to stay and deliver.

Following Table 1 depicts the four quadrants of today's Indian IT scenario indicating the changing times and changing preferences.

\begin{tabular}{|c|c|}
\hline Changing Preferences of next gen employees & Changing Technological Advancement \\
\hline 1. More Social, Collaborative & 1. Cloud-based digital learning environment \\
\hline 2. Need Anytime, Anywhere Learning & 2. Integrated learning solution \\
\hline $\begin{array}{l}\text { 3. Low attention \& retention span, differential } \\
\text { learning \& performing styles }\end{array}$ & $\begin{array}{l}\text { 3. Technical competency framework, linking roles, } \\
\text { competency \& learning/training needs }\end{array}$ \\
\hline Changing Business Performance Matrix & Changing Client \& Customer Preferences \\
\hline $\begin{array}{l}\text { 1. Career growth linked to learning, } \\
\text { development } \\
\text { 2. Employee engagement \& attrition control } \\
\text { 3. Operational excellence through faster } \\
\text { deployment of skills resources }\end{array}$ & $\begin{array}{l}\text { 1. Cost-effective, scalable, value added solution } \\
\text { 2. Learning on-the-go to deliver more with less } \\
\text { 3. Better customer experience through thought } \\
\text { partnership, agile \& learn approach }\end{array}$ \\
\hline
\end{tabular}

Table 1: Changing Preferences with Changing Time

Thus, it's realised that with changing times, preferences of employees, management, customers are rapidly changing, with apt support of next gen science and technology. Employees at the workplaces today are millennial with distinct preferences of likes and dislikes, learning styles, span of attention and retention, needs and wants, digital acumen, and social collaborations. They along with current generation need to work in cohesion and hence a combination approach is needed to make learning effective and impactful. On the other hand, due to such tech-savvy workforce in Indian IT organisations, the learning ecosystem and network also need to be next gen learner friendly with features like flexibility, cloud-based digital learning environment, end-to-end learning management system with appropriate mapping of roles 
and required competencies and hence learning interventions needed thereof etc. Indian IT management also have changed preferences for profitability, growth, employee engagement and retention, operational efficiency etc. and global IT customers also need a seamless customer experience with learning on-the-fly approach from their vendor partners, dynamic solution alignment and thought partnership with value orientation. All these changing aspects have varying impact on training and learning effectiveness, thus needing a detailed study thereof.

The purpose of this paper therefore is to examine such newer learning channels, meant specifically for this next generation workforce, and address the challenges \& opportunities and the various ways to build a stronger learning ecosystem to make the desired learning impact on ground on sustainable basis.

\section{Literature Review}

A successful learning program is one which has higher effectiveness \& efficiency towards expected business outcomes. Borate et al. (2014) inferred that training effectiveness can be evaluated with reference to four levels of training progress viz. reaction, learning, behavior and results (alongwith Kirkpatrick 4 level model) and change in each of these measures has positive impact on training results. Homklin et al. (2013) emphasized on self-efficacy, learning motivation, motivation to transfer and social support for prime factors responsible for training effectiveness. Fischer and Ronald (2011) explained that open-mindedness is a significant variable of training effectiveness and training become more successful if the participants and trainer work with progressiveness. Zaciewski (2001) examined employee' individual characteristics such as motivation, attitude, and basic ability, which affect a training program and its potential success. Saks and Haccoun (2007) consider learning transfer as the generalization of knowledge and skills learned in training on the job and the maintenance of acquired knowledge and skills over time. However, not much literature is available on ways and means of making any learning and training effective, tools, templates \& accelerators developed for the same and impact analysis thereof. Following model (6-D) represents a holistic method of building a wholesome approach towards end-to-end learning effectiveness. The model is depicted as per Fig 1 below.

Fig 1 - The Sixth Discipline of Breakthrough Learning

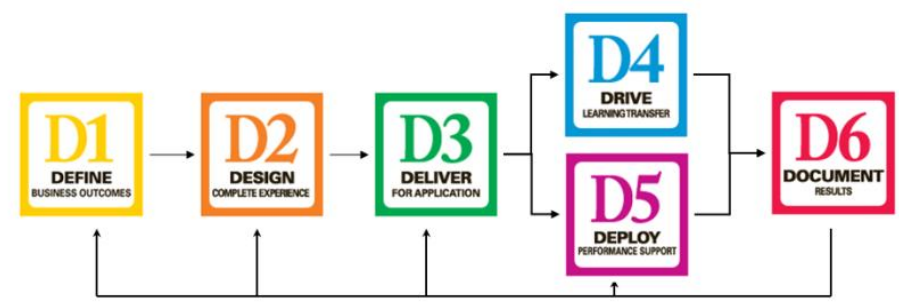

(Source: The Six Discipline of Breakthrough Learning by Roy V. H. Pollock, Andy Jefferson, Calhoun W. Wick)

This 6-D breakthrough learning model covers the end-to-end learning and transfer of learning process for it eventual success. For an IT industry, training\& learning being an integral part of talent supply chain, it's very important that these learning channels are well defined, designed, developed, deployed to suit the changing needs and wants of next gen employees alongwith the other generation employees. At the same time, it's essential to measure learnability and progressive learning value of talent development so as to build short term and long term learning assets for the organisations.

Some of the prominent learning effectiveness models are Kirkpatrick's four level model, Philips ROI model, Balanced Score Card model, Hamblin's five level model, Warr's framework of evaluation, Virmani and Premila's model of evaluation, Peter Bramely‘s model of evaluation and David Reay's approach to evaluation.Catherine Lombardozzi (2016) suggested a 5 step iterative process for creating a learning environment. This include Envisioning (covering the purpose, context and components of learning environment), Finding (involving finding out the range of resources addressing the problem), Curating (needing experts judgement to design the components of learning environment to suit the higher purpose and specific categories of learners), Assembling (entailing collating and packaging the selected materials and activities, to make them available to learners effectively) and Cultivating (assessing impact of new design and implementation on talent development and making it current and fresh all the time)

\section{Learning Effectiveness for growth of an IT organisation- a Theoretical Construct}

As indicated earlier, effectiveness of any training \& learning program should be rightly measured based on how it impacts business results, at strategic as well as operational level. These business results can be analysed in terms of hard and soft 
data, both from employee perspective as well as management perspective. For a fully blown conceptual framework, a construct can be defined in terms of multiple variables that are simultaneously acting on each other as well as on ultimate outcome. Such variables could be independent, dependent, moderating or intervening in nature with a degree of correlation between them. The conceptual model, so defined, analyses the impact of the influencing variables such as profile of learners, relevancy of learning ecosystem and support from organisation and managers, on intervening variable like skill \& competency creation as learning value and eventually on dependent variables, like productivity, revenue growth \& profitability, employee attrition and engagement etc.

\section{Next Generation Learning - How does that look like?}

Next Generation Learning, though technology agnostic, is technology enabled to ensure effectiveness, efficiency and scale. Andrew Calkins and Kristen Vogt (2013) stated that, from learner's perspective, following are the key asks:

- Personalized to the ways I learn best

- Flexible so that I can try different ways to learn

- Interactive and engaging so that I participate in the learning

- Relevant to the life I'd like to lead

- Organized around my own progress against goals I understand

- Constantly informed by different ways of demonstrating and measuring my progress

- Collaborative with teachers and peers, unlimited by proximity

- Agile and supportive when I need extra help

- Challenging but achievable, with opportunities to become expert in an area of interest

- Available to me as much as it is to every other learner

\section{Dimensions of Next Gen Learning}

Next gen learning will take root more broadly than it is today when we focus on six dimensions. The key factors are as per fig 2 below.

Fig 2 - Sixth Dimensions of Next Generation Learning

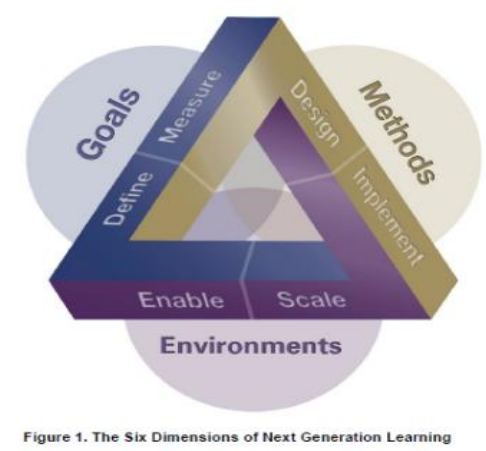

(Source: Andrew Calkins and Kristen Vogt (2013), Next Generation Learning: The pathway to Possibility)

These dimensions are : Define (for deeper and more comprehensive outcomes, indicating readiness), Measure (for formative and summative use), Design (that is personalized, competency-based, agile, easily accessible, and enabled by the deeply integrated use of technology), Implement(encompassing restructuring of roles, budgets, schedule, resources, infrastructure support etc.), Enable (internally and externally) and Scale (for rapid rollouts addressing change management, openness and cost effectiveness etc.). Thus, indicating that there is a need to redefine and rebuild the newer ways of learning network and learning ecosystems to suit next generation learners and next generation learning, with specific reference to service sector such as IT.

\section{Building a next gen Learning Ecosystem for an IT industry - Objectives and Benefits}

Today's business ecosystem operates in VUCA (Volatile, Uncertain, Complex and Ambiguous) world, and hence traditional strategies and business approaches will not work in making Indian organisations future ready and globally competitive. Leadership \& Talent Development professionals have to innovate and build newer models and delivery channels not just to sustain but excel in the business for sustainable profitable growth, they need to be disruptive in thoughts and actions. The next generation ecosystem, therefore, need to address some of the pressing asks e.g.: 
- Breaking the traditional mould of learning and talent development, understand and analyse different types of innovations and creations, practice sharing and gaining, prototyping and walk-throughs.

- Social and Collaborative learning leadership at all level, operational as well as strategic, addressing diversity \& inclusion, people management for transformations, co-creation, co-innovation etc.

- Building client-centric, growth oriented learning culture for enterprising mind-set through learning, acquiring and delivering the results for delivery \& operational excellence.

When one looks at the cross section of the next gen learners, it's seen that there is a major shift in the learning patterns ofnext gen employees. There are challenges in retaining what is learnt and in motivating Gen Y to improve the cycle of learning among employees. Creating a learning culture among corporations has never been so challenging in past. This has resulted into companies thinking about building a highly competitive learning model to take care of challenges such as multi-generation force working together, reduced attention and retention span among employees, experiential learning, in place of classroom learning, teaching has given way to facilitation and coaching, the time is of information overdose and rapid advances of technology, higher attrition and lower productivity etc. This leads to a need for innovative and creative learning model. According to Kolb, an influential researcher learning is cyclical with 3 key stages such as sending \& feeling, watching \& reflecting, and thinking \& doing. This, therefore, needs some innovative and creative learning strategy, plan and execution which should primarily addresses matters such as empowered workgroup sessions, advances of technology \& systems, hybrid learning model, deployment of social and collaborative ideas, focus more experiential learning with development happening more on ground with managers supporting and motivating them periodically etc. Such a next gen learning ecosystem:

- Is intuitive, transparent, and specifically tailored to their needs based on culture and growth of individual and organisations

- Accessible at all times whether online, offline, other devise or mobile, supplies the information needed within a few clicks, has a workflow that logically follows their own thinking, it should be flexible, collaborative, social, interactive and engaging, has adjustable gears to have own-pace learning

- Looks and feels familiar, like the web technology used every day, interact with others for sharing of experience and information. It should be relevant, responsive and supportive

- It should be challenging but achievable and is available anytime, anywhere and for any body

It's therefore important for L\&D professionals to build an outcome oriented learning ecosystem that aims for best-in class employees, bridge the talent gaps with required resource strength across the organisation, learning to be considered as a means to develop competitive competencies for sustainable growth and hence receive consistent funding from sponsors and management. Learning Organisations should spend more time, efforts and money on strategic purposes rather than just operational purposes, as this will help build the needed learning ecosystem for required business impact. In order to achieve such strategic orientation, L\&D organisations need to study company's financials and business model well, work closely with business and operational leadership teams to convert strategy into actions, focus on end-to-end learning cycle i.e. from identification of learning needs until impact analysis \& beyond, and network outside the given organisation such as those with researchers, vendors, conferences, journals etc. to understand market dynamics and outside in views. A journey of moving from operational to strategic include:

- From Instructor led training to on-the job learning, from in-person training to virtual learning

- From classroom training to anytime, anywhere learning, for higher adaptability

- From reinforced training to mentor-supported, blended learning for better impact

- From static, fixed device training to video-based, interactive, mobile, any device learning

- From fixed duration, fixed schedule formal training to self-paced, flexible informal learning

- From traditional closed content training to gamified, open source (MOOCs, YouTube) learning, collaborative learning through social media networks etc. for better team cohesion

- From Macro learning to micro, bite-sized Nano Learning for easier absorption and assimilation

As the new world realities are changing on continual basis, professionals and industry experts need to understand, appreciate and embrace the same for early adaption. The primary objectives of such next gen learning ecosystem, therefore is:

- To enable Anytime, Anywhere learning, aligned to the business needs and wants

- To empower higher, faster and cost-effective demand fulfilment through up-skilling \& cross-skilling

- To provide continuous learning opportunity for all employees equally well through any channel, using any devise and for any content

- To enable productivity improvement for operational excellence through accelerated learnings \& enhanced competencies 
- To promote a learning culture and a learning ecosystem, enabling higher employee engagement, learning ownership by learners and thus higher learnability \& skill quotient of the organization

In matured IT organisations, each learner is provided with a customised learning path to learn specific technology or functional skills, based on his/her background, current competencies (vis-à-vis expected competencies) and in accordance with the strategic skill inventory position or demand-driven fulfilment plan. Through a pre-configured learning landscape, these identified employees then begin their learning cycles consisting of structured digital contents, cloud-based lab sessions and specific assessments for the given proficiency level, to be completed in a given time. Their learning cycle time is monitored in a Learning Management System (LMS) and so is the result of their evaluations. The Learning Index which has a correlation with these two variables, is then calculated for each learner for assessing his/her learning productivity.

Additionally, IT organisations also needs to involve this global workforce in idea generations, decision making, implementation of initiatives and making change happen on ground. A crowdsourcing initiative which addresses the above objectives for building a client centric organisation, also need to be an integral part of the next gen learning ecosystem. These ideas are expected to help organisation generate employee brainwaves for pioneering and ingenious ideas to deliver customer value and business value. Newer learning ecosystems are therefore expected to tap such diverse ideas from employees, in different business fields such as sales \& marketing, corporate strategy \& brand building, talent sourcing \& development, delivery\& operational excellence etc. These initiatives, their implementations and learning thereof are expected to benefit the organisation for employee retention, higher engagement for co-creating the software assets and co-branding the innovative solutions accordingly.

Traditional libraries also need to get transformed into digital learning centres to accommodate digital e-libraries, leadership \& technical videos, short motivational clips, quick audio-video bytes, corporate membership with different digital and analogue libraries, deployment of new concepts such as bring your own devise (BYOD) and own learning (BYOL) and so on.

It's also central that next gen learning enabled is sticky and sustainable. The various factor needing consideration for such sticky digital learning ecosystem include:

- E-Learning movements - the next Gen employees need variety of content and solution in this field, such as new LMS, new cloud-based learning path and facilities, new Programming Interface Experience (PIE), automation, MOOCs, personalised learning experience (PLE), gamification, Big data, gamified learning, virtual learning, group learning, anytime learning etc.

- Learning steps include Content, Curation, Structure and Delivery, each of them is powerful step

- Learning measurement includes feedback on course and content (such as number of learning delivery days, coverage, new courses launched, took help etc.), Effective project performance management etc. metrics such as no. of channels, assessments, knowledge management etc.

\section{Challenges in implementing next gen learning ecosystem}

As we have 4-5 generations co-exist in a service sector like IT, the learning assimilation and transfer need to be consistent and sustainable. Change management should therefore be handled wisely and strategically. Special efforts need to be taken to promote and endorse the new ecosystem at all levels, all geographies and all functions within the organisation. A dedicated campaign is required to position the same strategically and provide high level on impetus, since inception. Teasers, danglers, promo-materials, quizzes to be aptly used to ensure that appropriate awareness, desire, knowledge building and abilities are getting developed and reinforced across classes and masses equally well. A governance model is required to be set up within the organisation, including senior management, sales and delivery organisations, HR and talent supply chain, inter-alia, employees at large. The reviews and evaluations should happen periodically to take stock of progress, challenges being faced, feed-backs received from stakeholders and progressive ways ahead.

\section{Innovative and Creative Practices for building a next gen Learning Ecosystem}

A next gen Learning ecosystem and robust yet flexible ecosystem is the true outcome of innovative \& creative thinking. It's a well-known fact that "Knowledge is Power" in service sector like IT and hence, having right knowledge, skill and attitude is a very important need of such business practices. Employee attrition, scarcity of required skills, logistical constraints and ever changing customer demands for "what's new?" make it necessary to build a unique learning and development solution which addresses Anytime, Anywhere, Any content, Any device, Anybody(5-A) learning aspects of learning ecosystem successfully and more so in a win-win manner. Such learning ecosystem thus provides strategic, tactical and operational advantages to the organisation through following innovative measures: 
- Making learning ecosystem relevant to type of business model, make it helpful and employee-savvy, and also make it real and practical

- Deploying 5-A learning model through digital connected cloud-based environment having digital content, device independent model, hands-on practice sessions using cloud-based labs and evaluation model for anybody and everybody of the organisation

- Design Thinking principles are interwoven for building "Zero Bench" and "Zero Lag" philosophy achieved through newer learning models

- Credit Points are awarded to employees outshining the bench marks which can be redeemed later for a Wow accreditation

- Social media to be leveraged extensively for campaigning and promoting such new dimensional learning programs to woo the employees, across strata

- Partnership model is be extended across the talent supply chain and learning value system to network the suppliers and customers equally, thus providing value engineering and lean management benefits from end-to-end perspective

- Learning is eventually being leveraged effectively to provide true business benefits such as productivity gains, higher operational efficiency, better customer satisfaction, higher employee engagement \& lower attrition and sustainable profitable growth of businesses and individuals

\section{Potential benefits of next gen Learning Ecosystem:}

Some of the prominent advantages of next gen learning ecosystem in an IT organisation are as follows:

- 5-A Learning Model with 24*7 Learning opportunity

- Instructor led Classroom training transforming into Virtual Learning, Anytime, Anywhere learning

- Customized learning paths can be assigned to learners, based on strategic skill inventory and/or demand based forecast, cutting edge technology programs are offered to learners through on-demand channel

- Content learning, hands-on practice and skill assessments can be provided through digitized cloud model and brought to closure in time - Accelerated billability and Career progression

- Learners get opportunity to work on live or Center of Excellence projects through crowdsourced initiatives

- The entire learning cycle can be automated from nomination to assessments and eventual deployment, thus tracking the learnability and learning value as measure of learning investment

- Sowing the seeds of learning and performing organization, leveraging learning for high impact business performance

\section{Research Methodology}

A robust research methodology was deployed as part of the research. This is in light of the fact that the study revolved mainly around next gen employees, their specific needs \& wants and learning support that is required to enhance the learning effectiveness, to make positive impact on business results of Indian Information Technology organisations. Research was conducted across major metropolis in India, with specific focus on cities with IT concentration. Respondents were selected across varied age and experience groups, background, roles \& responsibilities in order to avoid any bias thereof. Sample size was selected at 714, a customised questionnaire was developed post detailed piloting with SMEs across sector. Reliability and Validity tests were conducted for appropriateness of the instrument, with required content, construct and criterion validity

\section{Result and Discussions}

A detailed descriptive and statistical analysis suggested that there is a significant correlation between the new age learning ecosystem (in terms of its modern design, suitability to new gen learners, digitised way of deployment and sustenance, promotion and adoption, assessment and evaluation for effectiveness etc.), learnability and business impact. Following fig indicates the impact of background of learners, as above on Business Impact. The scale used for analysis was Likert's 5 point scale. 
Fig 3 - Impact of Learning Ecosystem on Learnability and Business Impact

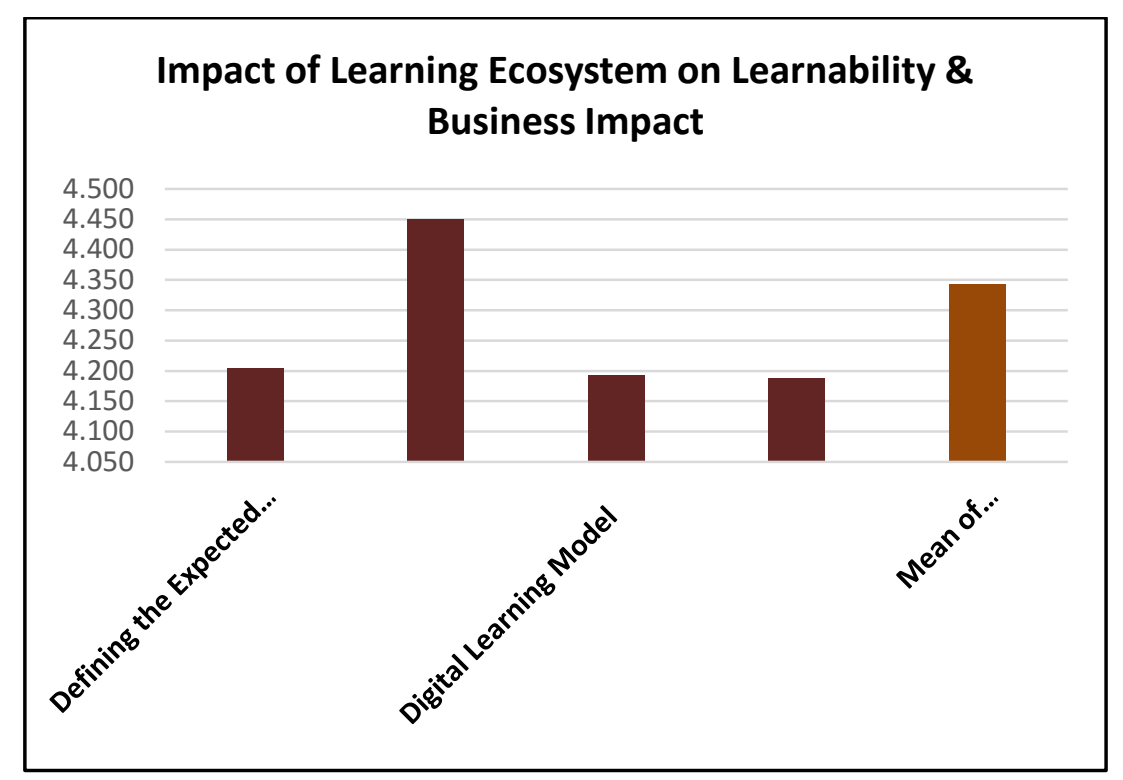

It's observed that $86.8 \%$ of the respondents perceive that defining right expectation of performance level prior to learning event is essential while $93.5 \%$ respondents are of the opinion that right learning environment is essential in terms of infrastructure, access, recording, LMS etc. About $83.3 \%$ of the respondents expressed that digital learning model is essential for higher learning outcome \& output such as e-learning, virtual learning, with anytime/anywhere access etc. It's also interesting to note that about $85 \%$ of the sample IT professionals indicated that some kind of technical/functional assessment /evaluation is essential to check the level of proficiency before, during and after the learning event, so as to examine the impact of learning pre \& post the intervention. This is in line with the level 2 of Kirkpatrick model. Overall, about $92.2 \%$ of the respondents expressed their strong views that this variable viz. the deployment of digital, flexible and modern learning ecosystem is critical as integral element of the business impact learning model. As part of research, null \& alternative hypothesis were developed, either to accept or reject the same, post statistical tests. Non-parametric statistical tests (such as Kruskal Wallis, Spearman's coefficient of correlation etc.) were carried out at 5\% level of significance (95\% confidence interval) which suggested a significant correlation among the variables, indicating that the new generation learning network \& ecosystem plays a significant role in determining learning effectiveness for business impact in service sector organisations, with specific reference to IT sector.

\section{Conclusion and Recommendation}

The changing learning styles of next gen learners with varying attention \& retention span, needs blended learning models which are gamified, collaborative and have key features of anytime, anywhere learning. This experiential learning methods, with strong mentoring support helps in higher learnability leading to business efficacy. Building such next gen, employee friendly and futuristic learning ecosystem is therefore, the need of hour as it's expected to provide many more benefits beyond obvious, eventually developing multi-skilled employees to embrace the changing times. Change management is the key to address the same and make business as usual through such movement. This also provides social benefits in terms of better work-life balance and enhanced happiness quotient of the employees, as they keep learning, growing and progressing in their career, sphere of work and social life. This journey, however, should not end post implementation, but be converted into a continuous movement so as to add more technologies, domain and specific business needs in the scope, addressing the changing needs and wants. In order to improve the quality of services and returns from the investments, L\&D is expected to check "Learnability Score" as a measure of ability of learners to undergo the specific programs, learn \& retain the learning at a desired speed, demonstrate their competitive edge and proficiency level through a structured evaluation model and eventually transfer these skills and competencies on the job successfully.

"When young people develop basic leadership and collaborative learning skills, they can be a formidable force for change and journey is the reward" said Peter M. Senge in his "The Fifth Discipline". This cultural shift should pave way fora new beginning and a formidable change to stay and prosper. The next gen learning ecosystem should transformed the organisations into next phase of maturity wherein continuous learning \& its application on the job, for sustainable allinclusive growth of employees and business, is considered as the business as usual. 


\section{References}

[1] Andrew Calkins and Kristen Vogt (2013): Next Generation Learning: The Pathway to Possibility

[2] Aguinis, Herman and Kraiger, K. (2009). "Benefits of Training and Development for Individuals, Teams, Organizations and Society", Annual Review of Psychology, 60(4)

[3] Antonio Arago'n-Sa'nchez, Isabel Barba-Arago'n and Raquel Sanz-Valle (2003), "Effects of training on business results", International Journal of Human Resource Management 14(6), 956-980

[4] B.K. Punia, Saurabh Kant (2013), "A review of factors affecting training effectiveness vis-à-vis managerial implications and future research directions" 2(1), 6-8

[5] Beigi, Mina and Shirmohammadi, Melika (2011). "Effects of an Emotional Intelligence Training Program on Service Quality of Bank Branches", Managing Service Quality, 21(5)

[6] Birdi, Kamal S. (2005). "No Idea- Evaluating the Effectiveness of Creativity Training", Journal of European Industrial Training, 29(2)

[7] Fischer, R. (2011). "Cross-Cultural Training Effects on Cultural Essentialism Beliefs and Cultural Intelligence", International Journal of Intercultural Relations, 35(6)

[8] Haslinda, A. and Mahyuddin, M.Y. (2009). "The Effectiveness of Training in the Public Service", American Journal of Scientific Research, 6, 39-51

[9] Hema Mani (2011) - "Innovative Learning and Development Strategies"

[10] Kalemci, P. (2005). "General Overview of Training Effectiveness and Measurement Models", Journal of Commerce and Tourism Education Faculty, 1

[11] Kirkpatrick, D. L. (1976). Evaluation of Training, Training and development handbook: A guide to human resource development, New York; McGraw-Hill Company.

[12] Michael F. Gorman (2011). "A Case Study in Effectively Bridging the Business Skills Gap for the Information Technology Professional", Journal of Education for Business, 86, 17-24

[13] NASSCOM - The IT-BPM Sector in India, Strategic Review 2016 (2016), 1-184

[14] Roy V. H. Pollock, Andy Jefferson, Calhoun Wick - The Six Discipline of Breakthrough Learning

[15] Tassanee Homklin, Yoshi Takahashi \& Kriengkrai Techakanont (2013), Effects of Individual and Work Environment Characteristics on Training Effectiveness: Evidence from Skill Certification System for Automotive Industry in Thailand, International Business Research, 6(12), 1-16

[16] Zaciewski, R.D. (2001). "Measuring Training's Effectiveness”, Quality Progress, 34(6) 